\title{
Autopercepção e estado de saúde bucal de adolescentes puérperas
}

\author{
Constanza MARÍN ${ }^{1}$; Elisabete Rabaldo BOTTAN²; Jessica \\ Vavassori de FREITAS ${ }^{3}$; Naiara Helena GARLINI ${ }^{4}$ \\ 1 - Doutora em Periodontia. Curso de Odontologia da Universidade da Região de Joinville - UNIVILLE. Grupo de Pesquisa Aten- \\ ção à Saúde Individual e Coletiva em Odontologia/UNIVALI/CNPq; 2 - Professora vinculada ao Grupo de Pesquisa Atenção à \\ Saúde Individual e Coletiva em Odontologia/CNPq - Curso de Odontologia Universidade do Vale do Itajaí - UNIVALI; 3 - Doutora \\ em Odontologia Clínica; 4 - Cirurgiã-dentista.
}

\section{Resumo}

Objetivo: identificar se a autopercepção de saúde bucal de um grupo de adolescentes puérperas corresponde ao estado clínico. Materiais e métodos: estudo descritivo, do tipo transversal. A coleta de dados ocorreu pela aplicação de questionário estruturado e do exame clínico da cavidade oral. Resultados: participaram 80 adolescentes puérperas com idades entre $13 \mathrm{e}$ 17 anos. A maioria (59\%) não recebeu informações sobre saúde bucal e gravidez. A consulta odontológica durante a gestação foi efetuada por $50 \%$, tendo como motivo mais frequente $(67,5 \%)$ a consulta de rotina. A maioria $(70 \%)$ não utiliza fio dental e $52,5 \%$ referiram escovar os dentes três vezes ao dia. A condição de saúde bucal regular foi citada por $53 \%$, seguida pelo nível bom (35\%) e ruim (12\%). Acredita ser portadora de cárie foi citado por $55 \%$ e $71 \%$ não perceberam alterações na gengiva. O CPO-D médio do grupo foi de 2,8. O exame periodontal demonstrou que $59 \%$ apresentaram todos os sextantes saudáveis; $26 \%$ tinham, pelo menos, um sextante com sangramento; $5 \%$ com presença de cálculo dental e sangramento após sondagem; $10 \%$ com bolsa periodontal de 4 a $5 \mathrm{~mm}$. Houve uma relação significativa entre percepção de cárie e avaliação clínica $(\mathrm{p}=0,00)$ e entre CPITN e percepção da condição de saúde bucal $(\mathrm{p}=0,03)$. Conclusão: Apesar das aproximações entre percepção e dados clínicos não se pode afirmar que melhores valores de CPO-D e de CPITN estejam efetivamente relacionados a uma melhor percepção sobre saúde bucal.

PALAVRAS-CHAVE: Saúde bucal; Autopercepção; Adolescente; Gravidez. 


\section{Introdução}

A literatura relata que adolescentes gestantes podem sofrer mais mudanças no seu estado de saúde quando comparadas às gestantes de outras faixas etárias. As características fisiológicas e psicológicas da adolescência contribuem para com uma gestação de risco. A gravidez na adolescência, por estar associada a complicações no estado de saúde materna e do bebê, é considerada como um problema de saúde pública ${ }^{1,2}$.

Do ponto de vista odontológico, a adolescência é um período em que o risco de cárie dentária e de gengivite aumenta em decorrência de comportamentos displicentes para com a saúde bucal. Já, durante a gravidez, as alterações fisiológicas inerentes a esta fase, se associadas às modificações de hábitos de vida, podem determinar o aparecimento, ou agravamento, de doenças da cavidade oral, como cárie e doença periodontal ${ }^{3-6}$.

A conscientização sobre necessidade de cuidados para com a saúde bucal favorece a melhoria do estado de saúde em geral das pessoas. Neste sentido, o conhecimento sobre diferentes procedimentos de atenção à saúde bucal durante a gestação é de suma importância para o desenvolvimento e manutenção de hábitos saudáveis. O baixo nível de conhecimento contribui para com uma errônea percepção de suas reais condições de saúde ${ }^{4,7}$. Portanto, pesquisas envolvendo aspectos referentes à avaliação de indicadores clínicos e de percepção podem contribuir para com o planejamento de ações que, efetivamente, atendam às necessidades de grupos específicos.

Definiu-se, então, como objeto deste estudo identificar se a autopercepção de saúde bucal de um grupo de adolescentes puérperas corresponde ao estado clínico.

\section{Materiais e métodos}

Esta investigação caracteriza-se como um estudo descritivo, do tipo transversal, mediante levantamento de dados primários. 
O projeto de pesquisa foi submetido e aprovado pelo Comitê de Ética em Pesquisa da Univille sob o nº 197.629.

Participaram do estudo oitenta (80) adolescentes puérperas, provenientes da demanda espontânea de um hospital situado numa cidade do litoral norte de Santa Catarina, no período de agosto de 2012 a março de 2013. Todas as adolescentes que aceitaram participar da pesquisa assinaram o Termo de Consentimento Livre Esclarecido.

A pesquisa foi conduzida em duas etapas. A primeira constou da aplicação de um questionário, sob a forma de entrevista. $\mathrm{O}$ instrumento continha catorze (14) questões do tipo fechado, distribuídas em três campos. O primeiro abordando a caracterização da gestante (idade, escolaridade, renda); o segundo campo enfocava os cuidados odontológicos (hábitos de higiene oral e frequência da consulta odontológica). O terceiro campo referia-se à percepção sobre condições de saúde bucal (dentes e gengiva).

$\mathrm{Na}$ segunda etapa, foi realizado o exame clínico da cavidade oral, avaliando presença de cárie e doença periodontal. Para este exame, foram adotados os procedimentos definidos no levantamento epidemiológico nacional de saúde bucal, intitulado SB Brasil - Pesquisa Nacional de Saúde bucal ${ }^{8}$. Os exames clínicos foram realizados no leito hospitalar, com o auxílio de uma fonte de luz externa, por um único examinador, que foi previamente treinado.

A avaliação da presença de cárie foi efetuada através do índice CPO-D, cujo valor corresponde à soma do número de dentes cariados, perdidos e obturados/restaurados em cada indivíduo, de acordo com a Organização Mundial da Saúde (OMS). O exame clínico constou de inspeção visual direta e indireta com espelho bucal $n \div 05$. Os resultados foram anotados em um odontograma. Para fins de análise, o dente restaurado que não apresentava cárie foi considerado como saudável. 
No exame periodontal, foi medida a profundidade de sondagem avaliada pelo Índice Periodontal de Necessidade Tratamento (CPITN), o qual foi realizado com sonda periodontal modelo OMS. Para a determinação do CPITN, considerou-se a pior situação presente nos sextantes.

Os dados foram analisados com base na estatística descritiva, mediante cálculo da distribuição da frequência relativa, e posteriormente foi aplicado o teste não paramétrico do qui-quadrado, considerando como diferença significativa aquelas definidas por um "p" crítico igual ou menor que 0,05 .

\section{Resultados}

\section{Caracterização socioeconômica}

O perfil das características pessoais e sociodemográficas das 80 adolescentes puérperas indica que a idade média era de 16 anos, variando de 13 a 17 anos e a maioria $(55 \%$; n=44) possuía ensino fundamental. Com relação ao estado civil, a maioria $(63,75 \%$; $\mathrm{n}=51)$ declarou viver em união consensual e $94 \%(\mathrm{n}=75)$ afirmaram que a renda familiar era de até três salários mínimos.

\section{Cuidados odontológicos}

Metade do número de participantes $(n=40)$ afirmou ter efetuado consulta odontológica durante a gestação, tendo como motivo mais frequente a consulta de rotina $(n=27 / 40 ; 67,5 \%)$. A maioria do grupo ( $n=42 / 80 ; 52,5 \%)$ referiu que efetua a escovação dental por três vezes ao dia, mas $70 \%(n=56 / 80)$ não utilizam fio dental.

Quando questionadas se haviam recebido informações sobre aspectos relacionados à saúde bucal e gravidez, $59 \%(n=47 / 80)$ disseram não ter recebido. Dentre as que receberam orientações $(\mathrm{n}=33 / 80 ; 41 \%)$, a principal fonte foi o curso pré-natal $(\mathrm{n}=16 / 33$; $48,5 \%)$, seguido pelo dentista $(n=10 / 33 ; 30,3 \%)$ e médico e enfermeira $(n=7 / 33 ; 21,2 \%)$. 
Autopercepção sobre as condições de saúde bucal

Os dados sobre percepção das participantes quanto a sua saúde bucal indicam que a maioria considera a sua condição como regular, acredita ser portadora de cárie e não percebeu alterações na gengiva, durante o período gestacional (Tabela 1).

TABELA 1 • Percepção das participantes sobre condições de saúde bucal

\begin{tabular}{|c|c|c|}
\hline Itens avaliados & $\mathbf{N}$ & $\%$ \\
\hline \multicolumn{3}{|c|}{ Como classifica sua saúde bucal? } \\
\hline Boa & 28 & 35 \\
\hline Regular & 42 & 53 \\
\hline Ruim & 10 & 12 \\
\hline \multicolumn{3}{|c|}{ Acredita ter cárie, neste momento? } \\
\hline $\operatorname{Sim}$ & 44 & 55 \\
\hline Não & 36 & 45 \\
\hline \multicolumn{3}{|c|}{ A gengiva sangra à escovação? } \\
\hline $\operatorname{Sim}$ & 37 & 46 \\
\hline Não & 43 & 54 \\
\hline \multicolumn{3}{|c|}{ Gengiva apresentou mudanças na gestação? } \\
\hline $\operatorname{Sim}$ & 23 & 29 \\
\hline Não & 57 & 71 \\
\hline
\end{tabular}

\section{Avaliação clínica}

O CPO-D médio do grupo foi de 2,8. A composição deste índice foi de: $5,5 \%$ dentes cariados; $0,9 \%$ dentes perdidos; e $3,6 \%$ dentes restaurados. Ao se considerar apenas as gestantes que possuíam cárie, o número médio do CPO-D foi de 4,14.

O exame periodontal (CPITN) demonstrou que 59\% ( $\mathrm{n}=47 / 80)$ apresentaram todos os sextantes saudáveis; $26 \%(\mathrm{n}=21 / 80)$ tinham, pelo menos, um sextante com sangramento; $5 \%(n=4 / 80)$ com presença de cálculo dental e sangramento após sondagem; $10 \%(\mathrm{n}=8 / 80)$ com bolsa periodontal de 4 a 5 milímetros; e nenhuma apresentou bolsa periodontal com 6 milímetros ou mais. Apenas $27,5 \%(\mathrm{n}=22 / 80)$ apresentavam-se saudáveis, ou seja, com CPITN igual a zero e ausência de cárie. 
A tabela 2 apresenta, de forma sintética, os dados da avaliação clínica.

TABELA $2 \cdot$ Dados da avaliação clínica do grupo

\begin{tabular}{llllllllll}
\hline CPITN & \multicolumn{4}{c}{ CÁRIE } & & & \multicolumn{2}{c}{$\begin{array}{l}\text { CPITN Zero e } \\
\text { Sem cárie }\end{array}$} \\
\hline Zero & & Maior Zero & Sem & Com & & \\
\hline $\mathbf{N}$ & $\%$ & $\mathbf{N}$ & $\%$ & $\mathbf{N}$ & $\%$ & $\mathbf{N}$ & $\%$ & $\mathbf{N}$ & $\%$ \\
\hline 47 & 59 & 33 & 41 & 32 & 40 & 48 & 60 & 22 & 27,5 \\
\hline
\end{tabular}

Relação entre avaliação clínica e autopercepção

A tabela 3 apresenta a relação entre os dados sobre a percepção das gestantes, no momento da pesquisa, quanto a ter ou não cárie dental e os dados obtidos com a avaliação clínica. Identificouse uma relação significativa $(\mathrm{p}=0,00)$ entre percepção e avaliação clínica. No grupo que respondeu positivamente ser portadora de cárie ( $n=44 / 80$ ), pela avaliação, a doença foi confirmada na maioria destas gestantes $(n=35 / 44)$. No grupo que acreditava não possuir cárie ( $n=36 / 80)$, ao exame clínico detectou-se que a maioria ( $n=23 / 36)$, efetivamente, estava livre de cárie.

A relação entre diagnóstico de cárie e percepção da condição de saúde bucal não se mostrou significativa $(\mathrm{p}=0,35)$, muito embora se identifique uma tendência de aumento da frequência da presença de cárie nos grupos cuja percepção se classificava nos níveis regular e ruim (Tabela 4).

Através da avaliação clínica, foi confirmado que, dentre as 37 que afirmaram ter percebido sangramento gengival quando da escovação, em $56,75 \%(n=21 / 37)$ das adolescentes pelo menos um sextante apresentou sangramento.

A relação entre CPITN e percepção da condição de saúde bucal (Tabela 5) indica que a frequência de portadoras de CPITN zero é maior entre as que classificaram sua saúde bucal como boa. Esta relação foi significativa $(\mathrm{p}=0,03)$. 
TABELA 3 • Relação entre percepção das gestantes sobre ser portadora de cárie (Sim ou Não) e a da avaliação clínica para cárie (Com cárie ou Livre de cárie).

\begin{tabular}{llll}
\hline Percepção & Sim & Não & Total \\
\hline & $(n=44)$ & $(n=36)$ & $(n=80)$ \\
\hline
\end{tabular}

Av. Clínica

\begin{tabular}{lcccccc}
\hline & $\mathbf{n}$ & $\%$ & $\mathbf{n}$ & $\%$ & $\mathbf{n}$ & $\%$ \\
\hline Com cárie & 35 & 79,5 & 13 & 36,1 & 48 & 60 \\
\hline Livre cárie & 9 & 20,5 & 23 & 63,9 & 32 & 40 \\
\hline Total & 44 & 100 & 36 & 100 & 80 & 100 \\
\hline$p=0,00$ & & & & & &
\end{tabular}

TABELA 4 • Diagnóstico de cárie e percepção sobre condição de saúde bucal (SB)

\begin{tabular}{lcccc}
\hline Diagnóstico de Cárie & Sem & \multicolumn{3}{c}{ Com } \\
\hline Condição SB & $N$ & $\%$ & $N$ & $\%$ \\
\hline Boa $(n=28)$ & 13 & 46,4 & 15 & 53,6 \\
\hline Regular $(n=42)$ & 17 & 40,5 & 25 & 59,5 \\
\hline Ruim $(n=10)$ & 2 & 20,0 & 8 & 80,0 \\
\hline$p=0,35$ & & &
\end{tabular}

TABELA $\mathbf{5}$ · CPITN e percepção sobre condição de saúde bucal (SB)

\begin{tabular}{lcccc} 
CPITN & Zero & \multicolumn{2}{c}{ Maior Zero } \\
\hline Condição SB & $N$ & $\%$ & $N$ & $\%$ \\
\hline Boa (n=28) & 21 & 75,0 & 7 & 25,0 \\
\hline Regular (n=42) & 23 & 54,8 & 19 & 45,2 \\
\hline Ruim (n=10) & 3 & 30,0 & 7 & 70,0 \\
\hline$p=0,03$ & & & &
\end{tabular}

Dentre as gestantes que classificaram sua saúde bucal como boa $(\mathrm{n}=28 / 80)$, apenas $35,7 \%(\mathrm{n}=10 / 28)$ apresentavam a boca saudável, ou seja, livre de cárie e com CPITN zero. A classificação da saúde bucal como boa, estatisticamente, não estava relacionada à ausência de cárie e doença periodontal $(\mathrm{p}=0,22)$. 


\section{Discussão}

A autopercepção sobre as condições de saúde bucal é um forte aliado para o alcance de níveis positivos de saúde bucal ${ }^{1,2,9,10}$. Estudos abordando autopercepção de condições de saúde bucal e necessidades de tratamento com grupos de adolescentes gestantes ainda são escassos 9 .

É necessário que as pessoas recebam orientações de modo adequado a fim de que sejam capazes de perceber a real condição de sua saúde. Neste sentido, é recomendada a efetivação da consulta odontológica durante o período gestacional, lembrando de que o atendimento odontológico não deve se limitar aos procedimentos clínicos; ele deve, também, contemplar orientações qualificadas ${ }^{5-7}$.

Neste estudo, identificou-se que apenas $50 \%(n=40 / 80)$ das participantes haviam realizado consulta odontológica durante a gravidez e 59\% ( $n=47 / 80)$ disseram não ter recebido orientações sobre saúde bucal no período gestacional. A alta frequência de não efetivação da consulta odontológica durante a gestação é abordada em diversos estudos 5 5-7,10,11. Quando a consulta odontológica é negligenciada, há uma maior probabilidade de agravamento de doenças da cavidade bucal que podem, inclusive, interferir na saúde geral da gestante e de seu bebê.

Não efetivar consulta odontológica durante o período gestacional está associado a fatores como dificuldade para acessar os serviços de saúde, desconhecimento sobre a necessidade do acompanhamento odontológico, crenças, medo, baixa percepção sobre a importância da higiene oral, dentre outros ${ }^{1,2,5,10}$. Assim, é fundamental que os serviços de saúde considerem todos estes fatores e planejem estratégias para melhorar o acesso à atenção odontológica nesta fase.

Nesse estudo, a maioria das participantes considerava sua condição de saúde bucal como regular, acreditava estar com cárie, uma baixa frequência identificou alterações na sua gengiva durante a gestação e a maioria não percebeu sangramento 
gengival quando da escovação. Alto índice de autopercepção sobre problemas dentais e baixo índice de autopercepção sobre problemas gengivais também foram identificados por Saliba et al. ${ }^{9}(2014)$, em pesquisa com adolescentes gestantes.

Com relação à cárie dental, os dados da avaliação clínica confirmaram que maioria das pesquisadas apresentava cárie dental. A literatura relata que, durante a gravidez, é frequente o aumento da incidência de cárie, geralmente associado a mudanças de hábitos alimentares, aumento da acidez na boca e descuido da higiene oral ${ }^{3,4,10,12}$.

Entre as adolescentes puérperas que participaram desse estudo, apenas $29 \%$ afirmaram perceber alguma alteração na gengiva, no entanto, $46 \%$ reportaram sangramento gengival à escovação. Estes dados evidenciam uma inconsistência, que nos leva a questionar se efetivamente estas adolescentes têm conhecimento suficiente para avaliar o que seja uma alteração gengival. Pela avaliação clínica simplificada, o sangramento gengival foi confirmado em $41 \%$ da amostra, percentual que está um pouco aquém daquele obtido pela percepção.

Em nosso entender, duas possíveis explicações para o fato do baixo índice de percepção sobre alterações na gengiva podem ser apresentadas. A primeira atribuímos aos achados clínicos os quais revelaram que $59 \%(n=47)$ possuíam todos os sextantes saudáveis, ou seja, um expressivo número de participantes apresentava gengiva saudável. A segunda hipótese é a de que, provavelmente, aquelas que, clinicamente, apresentaram alterações, como sangramento gengival, devem acreditar que esta é uma condição tida como normal, durante a gravidez, em decorrência de alterações hormonais.

A percepção de sangramento gengival não denotou uma maior procura pelo atendimento odontológico, uma vez que somente uma participante alegou ter consultado o dentista por causa do sangramento gengival. Acredita-se que muitas gestantes não interpretam o sangramento gengival como um sinal de inflamação, 
ou, ainda, que não seja um problema que necessite de atenção ${ }^{13}$. É oportuno destacar que a gravidez não causa gengivite, mas é um fator agravante, quando na presença de biofilme $e^{4,6}$.

A incidência e a severidade da gengivite em adolescentes recebem influência de diversos fatores, como: biofilme, cárie, respiração bucal, apinhamento dental. Porém, o aumento abrupto do nível hormonal apresenta um efeito transitório na inflamação gengival. A gengivite associada à puberdade pode apresentar diversas características clínicas da gengivite induzida por placa, com uma propensão maior a desenvolver sinais da inflamação na presença de pequenas quantidades de placa ${ }^{14}$.

A inflamação gengival em adolescentes puérperas pode ser considerada circunstancial. A gengivite pré-existente parece ser um fator importante, que se pode agravar com a gestação, porém sem perda de inserção. Portanto, mesmo sendo a gengivite uma infecção bacteriana, ela envolve outros mecanismos multifatoriais e sofre influência de diversos fatores retentivos locais, hormonais, assim como do período da gestação ${ }^{15,16}$.

É importante entender que os hormônios podem influenciar a homeostasia dos tecidos periodontais, sendo importante um trabalho preventivo e educativo assim como pesquisas de alterações gengivais em grupos maiores de gestantes adolescentes, nos diferentes trimestres, a fim de elucidar como os hormônios exercem seus efeitos neste período ${ }^{15,16}$.

As condições clínicas do grupo avaliado, de modo geral, podem ser consideradas positivas. O CPO-D médio foi de 2,8, que é inferior ao CPO-D médio nacional ${ }^{8}$ que era 4,25 e ao CPO-D médio da Região $\mathrm{Sul}^{8}$ cujo valor era de 4,01. Com relação ao CPITN zero, foi detectada uma situação muito próxima àquela do levantamento epidemiológico ${ }^{8}$. A presença de cálculo dental e sangramento após sondagem foi de $5 \%$ entre as pesquisadas enquanto a frequência apontada pelo SB Brasil 2010 era bem superior (28,4\%). O índice de bolsas rasas pelo SB Brasil 2010 era de $9 \%$ e neste estudo foi de $10 \%$. 
A doença periodontal pode variar desde um estado subclínico a alterações de cor e volume a um estado avançado de perda óssea. No entanto, a comparação com outros estudos epidemiológicos é difícil devido a pouca disponibilidade de trabalhos com adolescentes gestantes e aos diferentes critérios empregados para diagnóstico e definição de $\operatorname{casos}^{17}$.

Analisando-se a classificação quanto à percepção da condição de saúde bucal e os dados clínicos sobre CPITN e presença de cárie, identifica-se que o grupo das que consideravam ter uma boa condição de saúde bucal foi o que apresentou a maior frequência de CPITN zero e de ausência de cárie no momento da avaliação clínica. A frequência de CPITN maior do que zero e de cárie dental aumenta do nível regular para o ruim.

De modo geral, entende-se que a autopercepção da condição de saúde bucal se mostrou coerente com os achados clínicos, mesmo naquelas relações que não obtiveram significância estatística. Provavelmente, se a amostra fosse ampliada obteríamos uma confirmação da significância destas relações.

Independente dos resultados obtidos é fundamental ressaltar a necessidade de que os programas de atenção à saúde de gestantes adolescentes adotem a educação em saúde como uma importante ferramenta para o alcance de melhorias da saúde bucal. E, quando do planejamento destes programas, devem ser considerados os fatores psicossociais culturais e econômicos, além dos aspectos biológicos e clínicos ${ }^{4,10}$.

\section{Conclusão}

Apesar das aproximações entre percepção e dados clínicos não se pode afirmar que melhores valores de CPO-D e de CPITN estejam efetivamente relacionados a uma melhor percepção sobre saúde bucal, isto porque temos uma limitação do número de participantes. 


\section{Referências}

1 - Bwalya CB, Sitali D, Baboo K Zulu JM. Experiences of antenatal care among pregnant adolescents at Kanyama and Matero clinics in Lusaka district, Zambia. Reproductive Health. 2018; 15: 124. https:/doi. org/10.1186/s12978-018-0565-9.

2- Dias ACG, Teixeira MAP. Gravidez na adolescência: um olhar sobre um fenômeno complexo. Paidéia. 2010; 20(45): 123-131. https://doi. org/10.1590/S0103-863X2010000100015.

3 - Kateeb E, Momany E. Dental caries experience and associated risk indicators among Palestinian pregnant women in the Jerusalem area: a cross sectional study. BMC Oral Health. 2018; 18(1): 170. https://doi. org/10.1186/s12903-018-0628-x.

4 - Marla V, Srii R, Roy DK, Ajmera H. The Importance of oral health during pregnancy: a review. Medical Express. 2018; 5:(mr18002): 1-6.

5 - George A, Johnson M, Blinkhorn A, Ajwani S, Bhole S, Yeo A, Ellis S. The oral health status, practices and knowledge of pregnant women in south-western Sydney. Aust Dent J. 2013; 58(1): 26-33. doi: 10.1111/ adj.12024.

6 - Bressane LB, Silva LNBC, Vieira JMR, Rebelo MAB. Oral health conditions among pregnant women attended to at a health care center in Manaus, Amazonas, Brazil. Rev Odonto Ciênc. 2011; 26(4): 291-6. doi:10.1590/S1980-65232011000400003.

7 - Marín C, Maçaneiro CAR, Bottan ER, Vavassori F. Percepção do atendimento odontológico: comparações entre grupos de gestantes adultas e adolescentes. Rev. Atenção Saúde. 2015; 13(46): 65-71. doi:10.13037/rbcs.vol13n46.3099.

8 - Brasil. Ministério da Saúde. Secretaria de Atenção à Saúde. Secretaria de Vigilância em Saúde. SB Brasil 2010: Pesquisa Nacional de Saúde Bucal: resultados principais. Brasília: Ministério da Saúde; 2012.

9 - Saliba Rovida TA, Saliba Moimaz SA, Lima DP, Saliba Garbin CA. Selfperception and oral health in pregnant adolescents. Oral Health Dent Manag. 2014; 13(3): 842-6

10 - Fontes LBC, Lima NS, Silva SMS, Santos LCB, Marques KMG, Correia MCB et al. Pregnancy in adolescence and oral health changes. Inter Ped Dent Open Acc J. 2018; 2(2): 125-127. doi: 10.32474/ IPDOAJ.2018.02.000135.

11 - Amorim BF, Costa JF, Costa EL. Percepção de primigestas adolescentes sobre saúde bucal. Rev de Pesquisa em Saúde. 2011; 12(2): 13-7.

12 - Hemalatha VT, Manigandan T, Sarumathi T, Aarthi Nisha V, Amudhan A. Dental considerations in pregnancy-a critical review on the oral care. J Clin Diagn Res. 2013; 7(5): 948-53. doi:10.7860/ JCDR/2013/5405.2986. 
13 - Keirse M, Plutzer K. Women's attitudes to and perceptions of oral health and dental care during pregnancy. J Perinat Med. 2010; 38(1): 3-8. doi: https://doi.org/10.1515/jpm.2010.007.

14 - Mariotti A, Mawhinney MG. Endocrinology of sex steroid hormones and cell dynamics in the periodontium. Periodontol 2000. 2013; 61(1): 69-88.

15 - Mealey BL, Moritz AJ. Hormonal influences: effects of diabetes mellitus and endogenous female sex steroid hormones on the periodontium. Periodontol 2000. 2003; 32: 59-81.

16 - Murakami S, Mealey BL, Mariotti A, Chapple ILC. Dental plaqueinduced gingival conditions. J Periodontol. 2018; 89(Suppl 1): S17-S27.

17 - Duque A, Macrini M, Raigoza JP, Álvarez LG. Prevalencia de pérdida de inserción periodontal en una muestra de adolescentes de 15-19 años en Medellín, Colombia. Rev CES Odont. 2015; 28(2): 35-46. 


\title{
Self-perception and state of oral health in puerper adolescents
}

\begin{abstract}
Objective: to identify whether the oral health self-perception of a group of puerperal adolescents corresponds to the clinical status. Materials and methods: descriptive, cross-sectional study. Data collection took place through the application of a structured questionnaire and clinical examination of the oral cavity. Results: 80 puerperal adolescents aged between 13 and 17 years participated. The majority (59\%) did not receive information about oral health and pregnancy. The dental consultation during pregnancy was performed by $50 \%$, with the most frequent reason $(67.5 \%)$ being the routine consultation. The majority (70\%) do not use dental floss and $52.5 \%$ reported brushing their teeth three times a day. The condition of regular oral health was mentioned by $53 \%$, followed by the good ( $35 \%$ ) and bad (12\%) levels. Believing to have caries was mentioned by $55 \%$ and $71 \%$ did not notice changes in the gums. The group's average CPO-D was 2.8. The periodontal examination showed that $59 \%$ had all healthy sextants; $26 \%$ had at least one bleeding sextant; $5 \%$ with dental calculus and bleeding after probing; $10 \%$ with 4 to $5 \mathrm{~mm}$ periodontal pocket. There was a significant relationship for the relationship between caries perception and clinical evaluation $(\mathrm{p}=0.00)$ and between CPITN and perception of oral health condition ( $\mathrm{p}=0.03)$. Conclusion: Despite the similarities between perception and clinical data, it cannot be said that better values of CPO-D and CPITN are effectively related to a better perception of oral health.
\end{abstract}

KEYWORDS: Oral health; Self-perception; Adolescent; Pregnancy.

\section{Como citar este artigo}

Marín C, Bottan ER, Freitas JV, Garlini NH. Autopercepção e estado de saúde bucal de adolescentes puérperas. Rev Odontol Bras Central 2021; 30(89): 195-208. DOI: 10.36065/ robrac.v30i89.1469 